were created according to previous study of Artim-Esen B et al. 2014.

Results 142 SLE patients with a mean age at diagnosis of 33.29 (13.53) and a mean time of disease evolution of 15.82 (10.56) years were evaluated. Mean SLEDAI score was 5.91 (5.6) and mean SLICC value 1.1 (1.46). Autoantibody frequencies are: ANAs 87.3\%, anti-dsDNA 36.62\%, anti-Sm 9.2\%, anti-RNP 3.5\%, aCL IgG/M 20.15\%, aß2M IgG/M 21.88\%, LA 26.27\%, anti-Ro $45.07 \%$ and anti-La 16.2\%.

Profile $n^{\circ} 2$ included SLE patients with anti-Sm/RNP positivity. Profile $\mathrm{n}^{\circ} 3$ included patients with anti-Ro/La positivity. Profile $\mathrm{n}^{\circ} 4$ included patients with aCL IgG/M or $\mathrm{aB} 2 \mathrm{M}$ IgG/ $\mathrm{M}$ or LA positivity. Profile $\mathrm{n}^{\circ} 5$ included patients who exclusively showed anti-DNA positivity. Profile $n^{\circ} 1$ included all patients excluded from the other profiles.

Profile $\mathrm{n}^{\circ} 1$ patients were diagnosed earlier and had a longer disease evolution, whereas Profile $n^{\circ} 5$ patients were diagnosed later and with shorter disease evolution.

We observed a significant association among hematological affection and high levels of anti-Ro $(\mathrm{P}<0.0001)$, anti-La $(\mathrm{P}=0.022)$ and anti-Sm $(\mathrm{P}=0.018)$ was observed.

Conclusions An association between the presence of anti-Ro/La and hematological affection, as well as high incidence of Sjögren syndrome in these subgroup of patients was described.

\section{P20 ABSTRACT WITHDRAWN}

\section{\begin{tabular}{|l|l}
\hline P21 INVESTIGATION OF POSSIBLE PATHOGENIC \\
\hline
\end{tabular} AUTOANTIBODIES IN MEMBRANOUS LUPUS NEPHRITIS}

${ }^{1}$ Filipa Farinha, ${ }^{2}$ Ruth J Pepper, ${ }^{1}$ Chris Wincup, ${ }^{2}$ Alan Salama, ${ }^{1}$ David A Isenberg, ${ }^{1}$ Anisur Rahman. ${ }^{1}$ Centre for Rheumatology, University College London, London; ${ }^{2}$ Centre for Nephrology, University College London-Royal Free Campus, London, UK

10.1136/lupus-2020-eurolupus.69
Background The pathogenesis of membranous lupus nephritis (MLN) is poorly understood. Our objectives were to investigate, in patients with MLN, the presence of antibodies to nucleosomes and histones, which have been linked to the pathogenesis of proliferative lupus nephritis (PLN); and antibodies against phospholipase A2 receptor (PLA2R), previously identified in patients with idiopathic membranous nephropathy (IMN).

Methods Single-centre study, using banked serum samples from patients with biopsy-proven lupus nephritis (LN). For each patient, we aimed to identify two paired serum samples - one at the date of renal biopsy (active nephritis) and one when LN was quiescent. Antibodies were detected with indirect ELISA. Positivity cut-offs were $20 \mathrm{U} / \mathrm{mL}$ for anti-nucleosomes and PLA2R, and $40 \mathrm{U} / \mathrm{mL}$ for anti-histones antibodies. Levels of antibodies in paired active and quiescent LN samples were compared with a Wilcoxon signed-rank test, and a KruskalWallis test was used to compare the levels between independent LN groups.

Results Forty-nine active serum samples (13 pure MLN, 22 PLN and 14 mixed) and 41 paired quiescent samples were tested for anti-nucleosome and anti-PLA2R antibodies; and 44 active (13 pure MLN, 17 PLN and 14 mixed) and 38 paired quiescent samples were tested for anti-histones antibodies. Anti-nucleosome antibodies were present in $98 \%$ of active-LN patients. Total median level was 190.70 (IQR 681), with no significant difference between the three groups $(p=0.730)$. During quiescent $L N$, levels dropped significantly in PLN but not in pure MLN patients (figure 1). Anti-histone antibodies were positive in $32 \%$ of active- $\mathrm{LN}$ patients, with no significant difference between groups $(\mathrm{p}=0.828)$; their levels showed a similar trend to that of anti-nucleosomes (figure 1). Antibodies to PLA2R were negative in all patients.

Conclusions Anti-nucleosome and anti-histone antibody levels did not reflect disease activity in MLN. Anti-PLA2R antibodies were absent in these patients. None of these antibodies is likely to be playing a preponderant role in MLN pathogenesis.
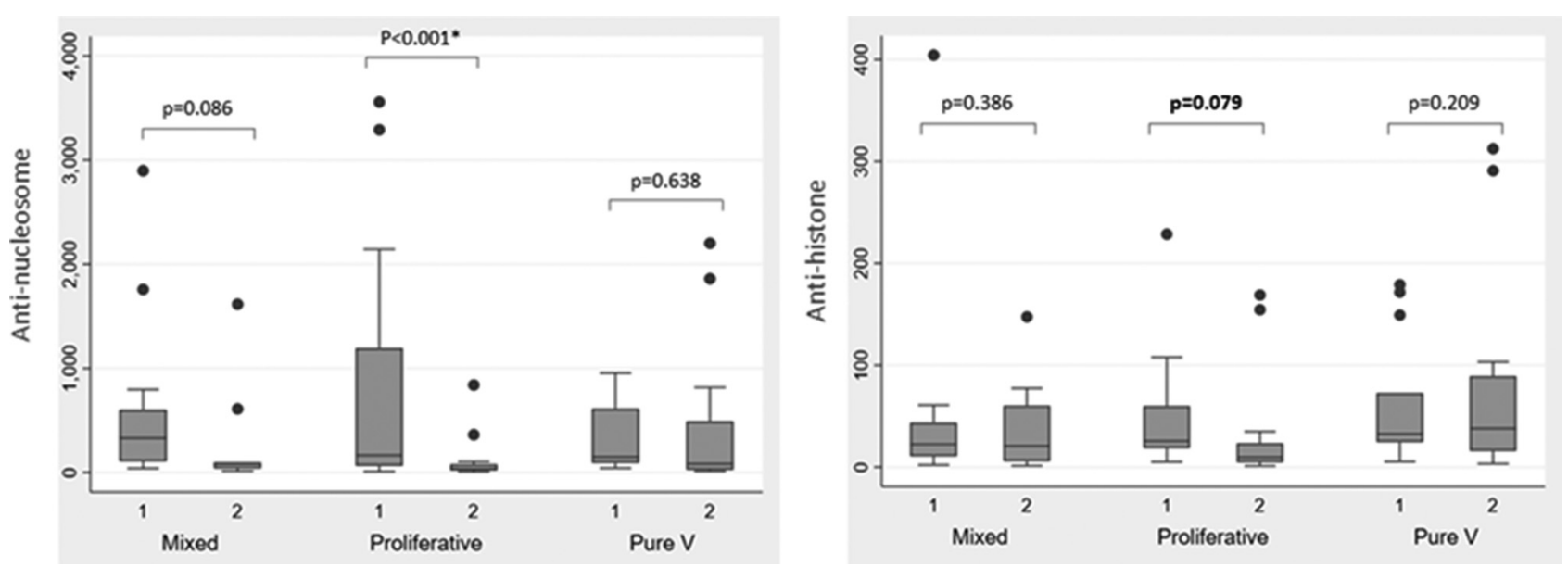

Abstract P21 Figure 1 Anti-nucleosome and anti-histone antibody levels (U/mL) in patients with mixed, proliferative and membranous $L N$, when the nephritis was active (1) and quiescent (2) 